\title{
SIGN-CHANGING CRITICAL POINTS FOR NONCOERCIVE FUNCTIONALS
}

\author{
YaOtian Shen — Zhouxin Li — Youjun Wang
}

\begin{abstract}
We study the existence of infinitely many sign-changing critical points and nonexistence of critical points to a class of noncoercive functionals.
\end{abstract}

\section{Introduction and main results}

This paper is concerned with the existence of sign-changing critical points to the problem which comes from the following functional

$$
I(u)=\int_{\Omega}\left[\frac{1}{2} a_{i j}(x) g^{2}(u) \partial_{i} u \partial_{j} u-F(x, u)\right] d x,
$$

where $\Omega$ is a bounded smooth domain in $\mathbb{R}^{n}, n \geq 2, a_{1}|\xi|^{2} \leq a_{i j}(x) \xi_{i} \xi_{j} \leq a_{2}|\xi|^{2}$, $a_{i j}=a_{j i}, a_{i j}(x) \in C^{\alpha}(\bar{\Omega})$, and $a_{2} \geq a_{1}>0$. The repeated indices indicate the summation from 1 to $N$

$$
\partial_{i} u=\frac{\partial u}{\partial x_{i}}, \quad F(x, u)=\int_{0}^{u} f(x, t) d t .
$$

If $g(u) \equiv 1$, then $I(u)$ is smooth. The results about existence of infinitely many critical points of $I(u)$ can be found in [1], [4] and the existence of infinitely many sign-changing critical points, in the case $a_{i j}(x)=\delta_{i j}$, was proved in [12].

2010 Mathematics Subject Classification. 35J60, 35J65.

Key words and phrases. Sign-changing, critical points, noncoercive functionals. 
If $g^{2}(u) \geq c>0$, then $I(u)$ is nonsmooth and its principal part is coercive. Results about the existence of critical points can be found in [2], [5]-[8], [10], [11], etc.

If $g^{2}(u)>0$, then $I(u)$ is nonsmooth and its principal part is noncoercive. There are seldom results about the existence of critical points of such kind of functionals. The first existence results are, up to our knowledge, due to [3], where the authors studied the existence of minimum point and other nontrivial critical points of the functional

$$
J(u)=\int_{\Omega}\left[\frac{|\nabla u|^{2}}{2(1+|u|)^{2 \alpha}}-\frac{|u|^{m}}{m}\right] d x
$$

proving that if $0<\alpha<N /(2 N-2)$ and $2<m<2^{*}(1-\alpha)$, then nontrivial critical points to $J(u)$ exist. Moreover, if $m \geq 2^{*}$ and $\Omega$ is a starshaped smooth domain, then the Euler-Lagrange equation of $J(u)$

$$
\begin{cases}-\operatorname{div}\left[(1+|u|)^{-2 \alpha} \nabla u\right]-\alpha(1+|u|)^{-(1+2 \alpha)}|\nabla u|^{2}=u^{m-1}, & x \in \Omega \\ u \geq 0, & x \in \Omega\end{cases}
$$

has no nontrivial solution in $H_{0}^{1}(\Omega) \cap L^{\infty}(\Omega) \cap H^{2}(\Omega)$.

As far as we know, there are no results on the existence of sign-changing critical points to nonsmooth functionals with coercive or noncoercive principal part.

The main purpose of the present paper is to study the existence of infinitely many sign-changing critical points and nonexistence of critical points of nonsmooth functionals with noncoercive principal part.

We note that the derivative of $I(u)$ is given by

$$
\begin{aligned}
& \left\langle I^{\prime}(u), \varphi\right\rangle \\
& \quad=\int_{\Omega}\left[a_{i j}(x) g^{2}(u) \partial_{i} u \partial_{j} \varphi+\frac{1}{2}\left(g^{2}(u)\right)^{\prime} a_{i j}(x) \partial_{i} u \partial_{j} u \varphi-f(x, u) \varphi\right] d x
\end{aligned}
$$

for every $\varphi \in C_{0}^{\infty}(\Omega)$.

In this paper, by introducing a change of variable, we transform the search of critical points $u(x)$ of (1.1) into the search of critical points $v(x)$ of the following functional:

$$
I_{1}(v)=\int_{\Omega}\left[a_{i j}(x) \partial_{i} v \partial_{j} v-F\left(x, G^{-1}(v)\right)\right] d x
$$

where $v=G(u), G(u)=\int_{0}^{u} g(t) d t, u=G^{-1}(v), g(t)>0$ in $[0,+\infty)$. We have $G^{-1}$ is of class $C^{1}$ and $I_{1}(v)$ is a smooth functional with coercive principal part.

We know that, for every $\psi \in C_{0}^{\infty}(\Omega)$,

$$
\left\langle I_{1}^{\prime}(v), \psi\right\rangle=\int_{\Omega}\left[a_{i j}(x) \partial_{i} v \partial_{j} \psi-\frac{f\left(x, G^{-1}(v)\right)}{g\left(G^{-1}(v)\right)} \psi\right] d x .
$$


We show that (1.2) is equivalent to (1.4), which means that $u$ is a critical point of $I(u)$ if and only if $v=G(u)$ is a critical point of $I_{1}(v)$. Indeed, if we choose $\varphi=\psi / g(u)$ in (1.2), then we immediately get (1.4). On the other hand, since $u=G^{-1}(v)$, if we let $\psi=g(u) \varphi$ in (1.4), we get (1.2). Therefore, in order to find the critical points of (1.1), it suffices to study the critical points of (1.3). Now, the Euler-Lagrange equation of the functional $I_{1}(v)$ can be simply write as

$$
-\partial_{j}\left(a_{i j}(x) \partial_{i} v\right)=\frac{f\left(x, G^{-1}(v)\right)}{g\left(G^{-1}(v)\right)}, \quad x \in \Omega .
$$

In this case, we can employ the sign-changing critical point theory for smooth functionals in [12] to study the existence of sign-changing critical points of $I_{1}(v)$, and then prove that those critical points are that of $I(u)$. If we assume that $g(u)$ is even and $f(x, u)$ is odd with respect to $u$, we have $v$ is odd and $v=G(|u|) \operatorname{sign} u$, thus $u$ has same sign of $v$.

REMARK 1.1. Since $G(u) \in H_{0}^{1}(\Omega)$, by Sobolev inequality, $G(u) \in L^{2^{*}}(\Omega)$. Assume that $\lim _{|u| \rightarrow \infty} G(u) /|u|^{\gamma}=c$, then, $u \in L^{\gamma 2^{*}}(\Omega)$. Let $g(u)=(1+|u|)^{-\alpha}$, if $\gamma 2^{*}>1$ then $\gamma=1-\alpha$, so, $\alpha<(N+2) / 2 N$.

In the following, we make the following assumptions on $f$ and $g$.

$\left(\mathrm{f}_{1}\right) f(x, u)$ is $\alpha$-Hölder continuous with respect to $x, u$, and there exists $1<p<2^{*}$ such that

$$
|f(x, u)| \leq C g(u)\left(1+|G(u)|^{p-1}\right)
$$

$\left(\mathrm{f}_{2}\right)|f(x, u)| \leq o(|g(u) G(u)|)$, as $u \rightarrow 0$.

$\left(\mathrm{f}_{3}\right)$ there exist $\mu>2, u_{0}>0$ such that for $|u| \geq u_{0}$,

$$
\mu F(x, u) g(u) \leq f(x, u) G(u) .
$$

$\left(\mathrm{f}_{4}\right) g(u) \in C^{\alpha}$ is even and nonincrease, $g(u)>0$ for $u \in[0,+\infty)$, and $f(x, u)$ is odd with respect to $u$.

REMARK 1.2. If $g(u)=1$, then $G(u)=u$, the above assumptions $\left(\mathrm{f}_{1}\right)-\left(\mathrm{f}_{3}\right)$ were used in [1], and in this special case

$\left(\mathrm{f}_{1}\right)|f(x, u)| \leq C\left(1+|u|^{p-1}\right)$.

$\left(\mathrm{f}_{2}\right) f(x, u)=o(|u|)$ as $u \rightarrow 0$.

$\left(\mathrm{f}_{3}\right) \mu F(x, u) \leq u f(x, u)$, for $\mu>2$ and $|u| \geq u_{0}$.

REMARK 1.3. By $\left(\mathrm{f}_{3}\right)$, for $|u| \geq u_{0}$, we have

$$
G(u)|G(u)|^{\mu} \frac{d}{d u}\left(|G(u)|^{-\mu} F(x, u)\right)=-\mu g(u) F(x, u)+G(u) f(x, u) \geq 0 .
$$

Then we can integrate inequality

$$
\frac{d}{d u}\left(|G(u)|^{-\mu} F(x, u)\right) \geq 0
$$


and obtain

$$
F(x, u) \geq C(x)|G(u)|^{\mu} .
$$

THEOREM 1.4. Assume that $\left(\mathrm{f}_{1}\right)-\left(\mathrm{f}_{4}\right)$ hold, then there exist infinitely many sign-changing critical points of the functional $I(u)$.

Corollary 1.5. Assume that $g(u)=(1+|u|)^{-\alpha}$ and $0<\alpha<1,2<m<$ $2^{*}(1-\alpha)$, then there exist infinitely many sign-changing critical points of the functional $J(u)$.

TheOrem 1.6. Assume $\left(\mathrm{f}_{1}\right),\left(\mathrm{f}_{3}\right)$ and $\left(\mathrm{f}_{4}\right)$ hold, then there exist infinitely many critical points of the functional $I(u)$.

Corollary 1.7. Assume that $g(u)=(1+|u|)^{-\alpha}$ and $0<\alpha<1,2(1-\alpha)<m<$ $2^{*}(1-\alpha)$, then there exist infinitely many critical points of the functional $J(u)$ with the corresponding critical values tending to $+\infty$.

If $a_{i j}(x)=\delta_{i j}$ and $f$ is independent on $x$, then the Euler-Lagrange equation of $I(u)$ is

$$
\begin{cases}-\operatorname{div}\left(g^{2}(u) \nabla u\right)+\frac{1}{2}\left(g^{2}(u)\right)^{\prime}|\nabla u|^{2}=f(u), & x \in \Omega, \\ u=0, & x \in \partial \Omega .\end{cases}
$$

THEOREM 1.8. Assume that $\Omega$ is a starshaped domain, and $f(u)$ satisfying

$$
|f(u)| \leq g(u)\left(C+(G(u))^{p^{\prime}}\right), \quad \text { for all } p^{\prime}>0,
$$

then if

$$
2^{*} \leq \frac{f(u) G(u)}{F(u) g(u)}
$$

problem (1.7) has no nontrivial solutions in $W^{2,2 n /(n+1)}(\Omega) \cap H_{0}^{1}(\Omega) \cap L^{\infty}(\Omega)$.

Corollary 1.9. Assume that $\Omega$ is a starshaped domain and

$$
F(u)=C|G(u)|^{\beta},
$$

then if $2^{*} \leq \beta$, problem (1.7) has no nontrivial solutions in $W^{2,2 n /(n+1)}(\Omega) \cap$ $H_{0}^{1}(\Omega) \cap L^{\infty}(\Omega)$.

In the case $g(u)=1$ and $G(u)=u$, the nonexistence result of Theorem 1.8 is well known to us. The result we obtained in Theorem 1.8 generalized this well known nonexistence result. An simple example in point is $g(u)=(1+|u|)^{-\alpha}$. In this case, if

$$
|F(u)|=C \frac{\left|(1+|u|)^{1-\alpha}-1\right|^{\beta}}{|1-\alpha|^{\beta}}
$$

and $2^{*} \leq \beta$, then conclusion of Theorem 1.8 holds. At this time, we see that $F(u) \sim C|u|^{(1-\alpha) \beta}$. This improve the results in [3]. 
REMARK 1.10. The assumption $m<2^{*}(1-\alpha)$ in Corollary 1.5 is necessary when $\Omega$ is a starshaped domain.

\section{Tools of even functional and Pohozaev identity}

In this section, we introduce some theorems which are needed in Section 3, in order to study the existence of infinitely many sign-changing critical points of $I(u)$. We improve the Pohozaev identity in the end of this section.

TheOREM 2.1. Let $X$ be a separable Hilbert space. $X_{i}$ be an $i$ dimensional subspace of $X$ and $Y_{i}=X_{i}^{\perp}$. Suppose $I(u) \in C^{1}(X, \mathbb{R})$ is even in $u$ and satisfies Palais-Smale condition, and if for all positive integers $i$, there exist $\rho_{i}$ and $r_{i}$ such that

(a) $b_{i}=\inf _{u \in Y_{i},\|u\|=r_{i}} I(u) \rightarrow+\infty$, as $i \rightarrow+\infty$.

(b) $a_{i}=\max _{u \in X_{i},\|u\|=\rho_{i}} I(u) \leq 0$.

Then $I(u)$ has a sequence of critical values tending to $+\infty$.

Proof. See [4].

Now we recall some facts from [12] which are needed in the proof of our main theorems.

Let $G \in C^{1}(E, \mathbb{R}), E$ is a Hilbert space, and the gradient $G^{\prime}$ be of the form

$$
G^{\prime}(u)=u-K_{G}(u),
$$

where $K_{G}: E \rightarrow E$ is a continuous operator. Let $\mathcal{K}:=\left\{u \in E: G^{\prime}(u)=0\right\}$ and $\widetilde{E}:=E \backslash \mathcal{K}, \mathcal{K}[a, b]:=\{u \in \mathcal{K}: G(u) \in[a, b]\}$. Let $\mathcal{P}$ be a positive cone of $E$. For $\mu_{0}>0$, define

$$
\mathcal{D}_{0}:=\left\{u \in E: \operatorname{dist}(u, \mathcal{P})<\mu_{0}\right\} .
$$

Then $\mathcal{D}_{0}$ is an open convex set containing the positive cone $\mathcal{P}$ in its interior. Set

$$
\mathcal{D}:=\mathcal{D}_{0} \cup\left(-\mathcal{D}_{0}\right), \quad \mathcal{S}=E \backslash \mathcal{D} .
$$

We assume that:

(A) $K_{G}\left( \pm \mathcal{D}_{0}\right) \subset \pm \mathcal{D}_{0}$.

Let $Y, M$ be two subspaces of $E$ with $\operatorname{dim} Y<\infty, \operatorname{dim} Y-\operatorname{codim} M \geq 1$ and $(M \backslash\{0\}) \cap(-\mathcal{P} \cup \mathcal{P})=\emptyset$; that is, the nontrivial elements of $M$ are sign-changing. We assume that $\mathcal{P}$ is weakly closed; that is, if $\mathcal{P} \ni u_{k} \rightarrow u$ weakly in $(E,\|\cdot\|)$, then $u \in \mathcal{P}$. Moreover, we assume that there is another norm $\|\cdot\|_{*}$ of $E$ such that $\|u\|_{*} \leq C_{*}\|u\|$ for all $u \in E$; here $C_{*}>0$ is a constant. We assume also that:

$\left(\mathrm{A}_{1}^{*}\right)$ Assume that for any $a, b>0$, there is $c_{2}=c_{2}(a, b)$ such that

$$
G(u) \leq a \quad \text { and } \quad\|u\|_{*} \leq b \quad \Rightarrow \quad\|u\| \leq c_{2} .
$$


$\left(\mathrm{A}_{2}^{*}\right) \lim _{u \in Y,\|u\| \rightarrow \infty} G(u)=-\infty$, and $\sup _{Y} G:=\beta$.

The following theorem was taken from [12, Theorem 5.6], readers can refer to [12] for its proof.

TheOREm 2.2. Assume $(\mathrm{A}),\left(\mathrm{A}_{1}^{*}\right)$ and $\left(\mathrm{A}_{2}^{*}\right)$. If the even functional $G$ satisfies the $\left(w^{*}-\mathrm{PS}\right)_{c}$ condition (see [12, Definition 3.3]) at level $c$ for each $c \in[\gamma, \beta]$, then

$$
\mathcal{K}[\gamma-\varepsilon, \beta+\varepsilon] \cap(E \backslash(-\mathcal{P} \cup \mathcal{P})) \neq \emptyset \quad \text { for all } \varepsilon>0 \text { small. }
$$

In the following, we introduce the Pohozaev identity in [9]. A main difference between our statement and the conclusions in [9] is that we consider the identity not in the usual $C^{2}$ space, but in the Sobolev space $W^{2, p_{1}} \cap H_{0}^{1}$, where $p_{1}=$ $2 n /(n+1)<2$.

We consider the following problem:

$$
\begin{cases}-\Delta u=f(u), & x \in \Omega, \\ u=0, & x \in \partial \Omega .\end{cases}
$$

TheOREM 2.2. Assume that $u$ is a solution to problem $(2.1), u \in W^{2, p_{1}}(\Omega)$ with $p_{1}=2 n /(n+1)<2$ and that

$$
|f(u)| \leq C\left(1+|u|^{q}\right),
$$

where $q \leq(n+1) /(n-3)$ if $n>3$ and $0<q<\infty$ if $n=3$. Then there holds

$$
-\frac{n-2}{2} \int_{\Omega}|\nabla u|^{2} d x+n \int_{\Omega} F(u) d x=\frac{1}{2} \int_{\partial \Omega}\left|\frac{\partial u}{\partial \nu}\right|^{2}\langle x, \nu\rangle d s,
$$

where $\nu$ denote the unit outer normal of $\partial \Omega,\langle x, \nu\rangle=\left\langle x_{i}, \nu_{i}\right\rangle$.

Proof. Since $u \in W^{2, p_{1}}(\Omega)$, we have $|\nabla u| \in L^{2 n /(n-1)}(\Omega)$. We can employ Hölder inequality to obtain that

$$
\int_{\Omega}|\Delta u||\nabla u| d x \leq\left(\int_{\Omega}|\Delta u|^{2 n /(n+1)}\right)^{(n+1) /(2 n)}\left(|\nabla u|^{2 n /(n-1)}\right)^{(n-1) /(2 n)} .
$$

Thus $(x \cdot \nabla u) \Delta u$ is integrable. On the other hand, since $u \in W^{2,2 n /(n+1)}(\Omega)$, we have $u \in W^{2-(n+1) /(2 n), 2 n /(n+1)}(\partial \Omega)$. Then we can use the fractional Sobolev embedding theorem to obtain that $u \in W^{1,2}(\partial \Omega)$. Multiplying $(2.1)$ by $(x \cdot \nabla u)$ and then integrating by part, we get

$$
-\int_{\Omega} \Delta u(x \cdot \nabla u) d x=\left(1-\frac{n}{2}\right) \int_{\Omega}|\nabla u|^{2}-\frac{1}{2} \int_{\partial \Omega}|\nabla u|^{2}\langle x, \nu\rangle d s .
$$

Furthermore, since $u \in W^{1,2 n /(n-1)}(\Omega)$, we obtain that $u \in L^{2 n /(n-3)}(\Omega)$ for $n>3$, and $u \in L^{q}(\Omega), 0<q<\infty$, for $n=3$. Then by (2.2),

$$
\int_{\Omega}|f(u)||\nabla u| d x \leq C\left(\int_{\Omega}|\nabla u||u|^{q} d x+\int_{\Omega}|\nabla u| d x\right),
$$


and by Hölder inequality,

$$
\int_{\Omega}|\nabla u||u|^{q} \leq\left(\int_{\Omega}|\nabla u|^{2 n /(n-1)}\right)^{(n-1) /(2 n)}\left(\int_{\Omega}|u|^{2 n /(n+1)} d x\right)^{(n+1) /(2 n)} .
$$

Since $q \leq(n+1) /(n-3)$, the index in the above inequality $q 2 n /(n+1) \leq$ $2 n /(n-3)$. It results that $f(u)(x \cdot \nabla u)$ is integrable and that

$$
\int_{\Omega} f(u)(x \cdot \nabla u) d x=\int_{\Omega} \frac{\partial F}{\partial x_{i}} x_{i} d x=-n \int_{\Omega} F(u) d x .
$$

This completes the proof.

\section{Proof of the main theorems}

In this section, $\|\cdot\|_{r}$ denote the norm of $L^{r}(\Omega), 1 \leq r<\infty$ and $C_{i}, i=0,1, \ldots$ will denote positive constants.

Let $E$ be the Hilbert space equivalent to $H_{0}^{1}(\Omega)$ and define the inner product of $E$ by

$$
\langle u, v\rangle=\int_{\Omega} a_{i j}(x) \partial_{i} u \partial_{j} v d x .
$$

The norm of $E$ is given by $\|u\|=\langle u, u\rangle^{1 / 2}$. That is, $E$ is the closer of $C_{0}^{\infty}(\Omega)$ under the norm $\|\cdot\|$.

Let $0<\lambda_{1}<\lambda_{2}<\ldots$ be the distinct eigenvalues of the operator

$$
-\partial_{j}\left(a_{i j}(x) \partial_{i} v\right)
$$

on $\Omega$ with zero boundary value. Then each $\lambda_{k}$ has finite multiplicity. The principal eigenvalue $\lambda_{1}$ is simple with a positive eigenfunction $\varphi_{1}$, and the eigenfunctions $\varphi_{k}$ corresponding to $\lambda_{k}(k \geq 2)$ are sign-changing. Let $N_{k}$ denote the eigenspace of $\lambda_{k}$. Then $\operatorname{dim} N_{k}<\infty$. We fix $k$ and let $E_{k}:=N_{1} \oplus \ldots \oplus N_{k}$. Let

$$
I_{1}(v)=\frac{1}{2}\|v\|^{2}-\int_{\Omega} F\left(x, G^{-1}(v)\right) d x, \quad v \in E .
$$

Then $I_{1}$ is of class $C^{1}(E, \mathbb{R})$ and

$$
\left\langle I_{1}^{\prime}(v), w\right\rangle=\langle v, w\rangle-\int_{\Omega} \frac{f\left(x, G^{-1}(v)\right)}{g\left(G^{-1}(v)\right)} w d x, \quad \text { for all } w \in E,
$$

that is, $I_{1}^{\prime}=\mathrm{id}-K_{I_{1}}$, where $K_{I_{1}}$ is a continuous operator.

Lemma 3.1. Assume that $\left(\mathrm{f}_{1}\right)$ and $\left(\mathrm{f}_{3}\right)$ hold, then $I_{1}(v)$ satisfies the (PS) condition.

Proof. Let $c \in \mathbb{R}$ and let $v_{h} \in H_{0}^{1}(\Omega)$ be such that

$$
I_{1}\left(v_{h}\right) \rightarrow c
$$


and

$$
\left\langle I_{1}^{\prime}\left(v_{h}\right), \varphi\right\rangle=\int_{\Omega}\left[a_{i j}(x) \partial_{i} v_{h} \partial_{j} \varphi-\frac{f\left(x, G^{-1}\left(v_{h}\right)\right)}{g\left(G^{-1}\left(v_{h}\right)\right)} \varphi\right] d x=o(1)\|\varphi\| .
$$

By $\left(f_{3}\right)$, we have

$$
F\left(x, G^{-1}\left(v_{h}\right)\right) \leq \frac{f\left(x, G^{-1}\left(v_{h}\right)\right) v_{h}}{\mu g\left(G^{-1}\left(v_{h}\right)\right)}+C_{0},
$$

where $C_{0}>0$ is a constant. Taking $\varphi=v_{h}$ in (3.2) and using (3.3), we obtain from (3.1) that

$$
\begin{aligned}
c \leftarrow \int_{\Omega}\left[\frac{1}{2} a_{i j}(x) \partial_{i} v_{h}\right. & \left.\partial_{j} v_{h}-F\left(x, G^{-1}\left(v_{h}\right)\right)\right] d x \\
& \geq \int_{\Omega}\left(\frac{1}{2}-\frac{1}{\mu}\right) a_{i j}(x) \partial_{i} v_{h} \partial_{j} v_{h} d x+o(1)\left\|v_{h}\right\|-C_{0}|\Omega|,
\end{aligned}
$$

where $|\Omega|$ denote the Lebesgue's measure of $\Omega$. This results that $v_{h}$ is bounded in $H_{0}^{1}(\Omega)$. Then there exists a subsequence of $v_{h}$, denote still by $v_{h}$, and $v \in H_{0}^{1}(\Omega)$ such that $v_{h} \rightarrow v$ in $H_{0}^{1}(\Omega)$ with $\left\|v_{h}-v_{k}\right\|_{p} \rightarrow 0$ as $h, k \rightarrow \infty$. We prove that $v_{h} \rightarrow v$ strongly in $H_{0}^{1}(\Omega)$. In fact, taking $\varphi=v_{h}-v_{k}$ in $\left\langle I_{1}^{\prime}\left(v_{h}\right)-I_{1}^{\prime}\left(v_{k}\right), \varphi\right\rangle$, through direct computation, we obtain that

$$
\begin{aligned}
\left\|v_{h}-v_{k}\right\|^{2} \leq & \int_{\Omega}\left|\frac{f\left(x, G^{-1}\left(v_{h}\right)\right)}{g\left(G^{-1}\left(v_{h}\right)\right)}-\frac{f\left(x, G^{-1}\left(v_{k}\right)\right)}{g\left(G^{-1}\left(v_{k}\right)\right)}\right| v_{h}-v_{k} \mid d x \\
& +o(1)\left\|v_{h}-v_{k}\right\| \\
\leq & \left(\int_{\Omega}\left|\frac{f\left(x, G^{-1}\left(v_{h}\right)\right)}{g\left(G^{-1}\left(v_{h}\right)\right)}-\frac{f\left(x, G^{-1}\left(v_{k}\right)\right)}{g\left(G^{-1}\left(v_{k}\right)\right)}\right|^{p /(p-1)} d x\right)^{(p-1) / p} \\
& \cdot\left\|v_{h}-v_{k}\right\|_{p}+o(1)\left\|v_{h}-v_{k}\right\|,
\end{aligned}
$$

here we have used Hölder inequality. On the other hand, from $\left(f_{1}\right)$, we have

$$
\int_{\Omega}\left|\frac{f\left(x, G^{-1}\left(v_{h}\right)\right)}{g\left(G^{-1}\left(v_{h}\right)\right)}\right|^{p /(p-1)} d x \leq C \int_{\Omega}\left(1+\left|v_{h}\right|^{p-1}\right)^{p /(p-1)} d x \leq C_{1} .
$$

Thus we get the conclusion from (3.4).

Before we prove Theorem 1.3, we need the following several lemmas which are similar to those in [12].

Lemma 3.2. $I_{1}(v) \rightarrow-\infty$ as $\|v\| \rightarrow \infty$, for all $v \in E_{k}$.

Proof. According to $\left(\mathrm{f}_{3}\right)$ and (1.6), we know that for $|v| \geq G\left(u_{0}\right)$,

$$
F\left(x, G^{-1}(v)\right) \geq C_{2}|v|^{\mu}, \quad \mu>2 .
$$

Thus for all $v$, we have

$$
F\left(x, G^{-1}(v)\right) \geq C_{2}|v|^{\mu}-C_{3},
$$


Furthermore, since $E_{k}$ is a finite dimensional space, and norms of a finite dimensional space are all equivalent, we have

$$
I_{1}(v)=\frac{1}{2}\|v\|^{2}-\int_{\Omega} F\left(x, G^{-1}(v)\right) d x \leq \frac{1}{2}\|v\|^{2}-C_{2}\|v\|_{\mu}^{\mu}+C_{3}|\Omega| \rightarrow-\infty,
$$

as $\|v\| \rightarrow \infty, v \in E_{k}$.

Now we consider another norm $\|v\|_{*}:=\|v\|_{s}$ of $E, s \in\left(2,2^{*}\right)$. Then $\|v\|_{s} \leq$ $C_{*}\|v\|$ for all $v \in E$; here $C_{*}>0$ is a constant and $\left\|v_{n}-v\right\|_{*} \rightarrow 0$ whenever $v_{n} \rightarrow v$ weakly in $(E,\|\cdot\|)$. Write $E=E_{k-1} \oplus E_{k-1}^{\perp}$. Let

$$
Q^{*}(\rho):=\left\{v \in E_{k-1}^{\perp}: \frac{\|v\|_{s}^{s}}{\|v\|^{2}}+\frac{\|v\|\|v\|_{s}}{\|v\|+D_{*}\|v\|_{s}}=\rho\right\},
$$

where $\rho, D_{*}$ are fixed constants. We have

LEMMA 3.3. $\|v\|_{s} \leq c_{1}$, for all $v \in Q^{*}(\rho)$, where $c_{1}>0$ is a constant.

By the assumptions, we may find a $C_{F}>0$ such that

$$
F\left(x, G^{-1}(t)\right) \leq \frac{1}{4} \lambda_{1}|t|^{2}+C_{F}|t|^{s}, \quad \text { for all } x \in \Omega, t \in \mathbb{R}
$$

here $2<s<2^{*}$. For any $a, b>0$, there is a $c_{2}=c_{2}(a, b)>0$ such that

$$
I_{1}(v) \leq a \quad \text { and } \quad\|v\|_{s} \leq b \quad \Rightarrow \quad\|v\| \leq c_{2} .
$$

By Lemma 3.2, $\lim _{v \in Y,\|v\| \rightarrow \infty} I_{1}(v)=-\infty$, where $Y=E_{k}$. Then $\left(\mathrm{A}_{1}^{*}\right)$ and $\left(\mathrm{A}_{2}^{*}\right)$ are satisfied.

We define $\sup _{Y} I_{1}:=\beta$. Denote $I_{1}^{\beta}:=\left\{v \in E: I_{1}(v) \leq \beta\right\}$. Let

$$
Q^{* *}:=Q^{*}(\rho) \cap I_{1}^{\beta}, \quad \inf _{Q^{* *}} I_{1}:=\gamma
$$

Set $\mathcal{P}:=\{v \in E: v(x) \geq 0$ for almost every $x \in \Omega\}$. Then $\mathcal{P}(-\mathcal{P})$ is the positive (negative) cone of $E$ and weakly closed. Similar to Lemma 5.4 in [12], there is a $\delta:=\delta(\beta)$ such that $\operatorname{dist}\left(Q^{* *}, \mathcal{P}\right):=\delta(\beta)>0$. We define

$$
\mathcal{D}_{0}\left(\mu_{0}\right):=\left\{v \in E: \operatorname{dist}(v, \mathcal{P})<\mu_{0}\right\}
$$

where $\mu_{0}$ is determined by the following lemma.

LEMMA 3.4. Under the assumptions of $\left(\mathrm{f}_{1}\right)-\left(\mathrm{f}_{3}\right)$, there exists a $\mu_{0} \in(0, \delta)$ such that $K_{I_{1}}\left( \pm \mathcal{D}_{0}\left(\mu_{0}\right)\right) \subset \pm \mathcal{D}_{0}\left(\mu_{0}\right)$.

Proof. The proof is quite similar to that of Lemma 2.29 in [12].

Let $\mathcal{D}:=-\mathcal{D}_{0}\left(\mu_{0}\right) \cup \mathcal{D}_{0}\left(\mu_{0}\right)$ and $\mathcal{S}:=E \backslash \mathcal{D}$. We assume

$$
Q^{* *}:=Q^{*}(\rho) \cap I_{1}^{\beta} \subset \mathcal{S} .
$$


Lemma 3.5. Assume that $\left(\mathrm{f}_{1}\right)-\left(\mathrm{f}_{4}\right)$ hold, then $I_{1}(v)$ has infinitely many signchanging critical points.

Proof. The proof is similar to that of Theorem 5.7 in [12].

Proof of Theorem 1.4. By Lemma 3.5, we know that there exist infinitely many sign-changing critical points of $I_{1}(v)$ and that the sign-changing critical point $v$ satisfies

$$
\int_{\Omega}\left[a_{i j}(x) \partial_{i} v \partial_{j} \varphi-\frac{f\left(x, G^{-1}(v)\right)}{g\left(G^{-1}(v)\right)} \varphi\right] d x=0, \quad \text { for all } \varphi \in E .
$$

Let $v=G(u)$, then $\partial_{i} v=g(u) \partial_{i} u$. Substituting it into the above equality, we get

$$
\int_{\Omega}\left[a_{i j}(x) g(u) \partial_{i} u \partial_{j} \varphi-\frac{f(x, u)}{g(u)} \varphi\right] d x=0, \quad \text { for all } \varphi \in E .
$$

Now let $\varphi=g(u) \psi, \psi \in C_{0}^{\infty}(\Omega)$, then

$$
\int_{\Omega}\left[a_{i j}(x) g^{2}(u) \partial_{i} u \partial_{j} \psi+\frac{1}{2}\left(g^{2}(u)\right)^{\prime} a_{i j}(x) \partial_{i} u \partial_{j} u \psi-f(x, u) \psi\right] d x=0,
$$

for all $\psi \in C_{0}^{\infty}(\Omega)$. Therefore, $u$ is a critical point of $I(u)$. Since $v=G(u)=$ $G(|u|) \operatorname{sign} u, v$ has same sign of $u$, thus $u$ is a sign-changing critical point of $I(u)$.

REMARK 3.6. If $\partial \Omega$ is smooth, then $v \in C^{2, \alpha}(\Omega)$.

Proof of Corollary 1.5. We need only to prove that $J(u)$ satisfies conditions $\left(\mathrm{f}_{1}\right)-\left(\mathrm{f}_{3}\right)$. In fact, since $m>2>2(1-\alpha)$, we can choose $\varepsilon>0$ such that $\mu=m(1-\varepsilon) /(1-\alpha)>2$, then there exists $u_{0}>0$ sufficiently large and such that for $|u| \geq u_{0}$,

$$
\mu F(u) g(u) \leq f(u) G(u)
$$

where

$$
F(u)=\frac{|u|^{m}}{m}, \quad f(u)=|u|^{m-1} \operatorname{sign} u, \quad G(u)=\frac{(1+|u|)^{1-\alpha}-1}{1-\alpha} \operatorname{sign} u .
$$

This implies that $\left(f_{3}\right)$ holds. Since $m>2$, it follows that $\left(f_{2}\right)$ holds also.

We employ Theorem 2.1 to prove Theorem 1.6.

Proof of Theorem 1.6. Theorem 2.1 applied. Firstly, we prove (a). We take $X_{i}=E_{i}=N_{1} \oplus \ldots \oplus N_{i}, Y_{i}=X_{i}^{\perp}$ and consider the following functional

$$
I_{1}(v)=\frac{1}{2}\|v\|^{2}-\int_{\Omega} F\left(x, G^{-1}(v)\right) d x .
$$

By $\left(\mathrm{f}_{1}\right)$ we know that $\left|F\left(x, G^{-1}(v)\right)\right| \leq C(1+|v|)^{p}$. Then for $v \in Y_{i}$, we have

$$
\|v\|_{2}^{2} \leq \frac{1}{\lambda_{i+1}}\|v\|^{2} .
$$


By Gagliondi-Nirenberg inequality $\|v\|_{p} \leq C_{p}\|v\|^{\alpha}\|v\|_{2}^{1-\alpha}$, where $C_{p}>0$ is a constant and $\alpha \in(0,1)$ is defined by

$$
\frac{1}{p}=\alpha\left(\frac{1}{2}-\frac{1}{N}\right)+\frac{1}{2}(1-\alpha)
$$

Then by (3.5), we get

$$
\|v\|_{p}^{p} \leq C_{p}^{p}\|v\|^{p \alpha}\|v\|_{2}^{p(1-\alpha)} \leq C_{p}^{p}\|v\|^{p \alpha} \lambda_{i+1}^{-p(1-\alpha) / 2}\|v\|^{p(1-\alpha)}=C_{p}^{p}\|v\|^{p} \lambda_{i+1}^{-p(1-\alpha) / 2} .
$$

Therefore, we have

$$
\begin{aligned}
I_{1}(v) & \geq \frac{1}{2}\|v\|^{2}-C_{p}^{p}\|v\|^{p} \lambda_{i+1}^{-p(1-\alpha) / 2}-C_{4}|\Omega| \\
& =\|v\|^{2}\left(\frac{1}{2}-C_{p}^{p}\|v\|^{p-2} \lambda_{i+1}^{-p(1-\alpha) / 2}\right)-C_{4}|\Omega| .
\end{aligned}
$$

Since $\lambda_{i} \rightarrow \infty$, we can take $\|v\|=r_{i}$ such that $C_{p}^{p} r_{i}^{p-2} \lambda_{i}^{-p(1-\alpha) / 2}=1 / 4$. Thus for $i \rightarrow+\infty$, we have $I_{1}(v) \rightarrow+\infty$.

Next we prove (b). By (1.6) we have $F\left(x, G^{-1}(v)\right) \geq C_{5}|v|^{\mu}$, for $|v| \geq G\left(u_{0}\right)$. Thus, for any $v$, we have $F\left(x, G^{-1}(v)\right) \geq C_{5}|v|^{\mu}-C_{6}$.

Since $X_{i}$ is a finite dimensional space, and norms of finite dimensional space are all equivalent, we deduce that

$$
I_{1}(v)=\frac{1}{2}\|v\|^{2}-\int_{\Omega} F\left(x, G^{-1}(v)\right) d x \leq \frac{1}{2}\|v\|^{2}-C_{5}\|v\|_{\mu}^{\mu}-C_{6}|\Omega| .
$$

This gives $\max _{v \in X_{i},\|v\|=\rho_{i}} I_{1}(v) \leq 0$, for $\rho_{i}$ sufficiently large. Since $g$ is even, we have $G$ is add and so does $G^{-1}(v)$, this results that $F\left(x, G^{-1}(v)\right)$ is even. Thus from Theorem 2.1, we get the conclusion of the theorem.

Proof of Corollary 1.7. Similar to the proof for Corollary 1.5, we obtain that $\left(f_{3}\right)$ holds. Then we can get the conclusion from Theorem 1.6.

Proof of Theorem 1.8. Assume on the contrary that $u \in H^{2}(\Omega) \cap H_{0}^{1}(\Omega) \cap$ $L^{\infty}(\Omega)$ is a solution of (1.7), then $v=G(u)$ is a solution of

$$
-\Delta v=\frac{f\left(G^{-1}(v)\right)}{g\left(G^{-1}(v)\right)}
$$

We multiply (3.6) by $v$ and integrate over $\Omega$. We have

$$
\int_{\Omega}|\nabla v|^{2} d x-\int_{\Omega} \frac{f(u) G(u)}{g(u)} d x=0 .
$$

Then (2.3) and (1.8) imply that $u \equiv 0$ on $\Omega$. 
Acknowledgements. The authors thank the referee for carefully reading this paper and suggesting many useful comments. The first and third authors were supported by NSF of China (No. 11371146 and No. 11201154) and the Fundamental Research Funds for the Central Universities (No. 2014ZZ0065). The second author was supported by NSF of China (No. 11201488) and Hunan Provincial Natural Science Foundation of China (No. 14JJ4002). Corresponding author: Zhouxin Li.

\section{REFERENCES}

[1] A. Ambrosetti And P.H. Rabinowitz, Dual variational methods in critical point theory and applications, J. Funct. Anal. 14 (1973), 349-381.

[2] D. Arcoya And L. Boccardo, Critical points for multiple integrals of calculus of variations, Arch. Rat. Mech. Anal. 134 (1996), 249-274.

[3] D. Arcoya, L. Boccardo L. Orsina, Existence of critical points for some noncoercive functionals, Ann. Inst. H. Poincaré Anal. Non Linéaire 18 (2001), 437-457.

[4] T. BARTSCH, Infinitely many solutions of a symmetric Dirichlet problem, Nonlinear Anal. 20 (1993), 1205-1266.

[5] M. Conti And F. Gazzola, Positive entire solutions of quasilinear elliptic problems via nonsmooth critical point theory, Topol. Methods Nonlinear Anal. 8 (1996), 275-294.

[6] M. Degiovanni And M. Marzocchi, A critical point theory for nonsmooth functionals, Ann. Mat. Pura Appl. 167 (1994), 73-100.

[7] Z.X. Li, Y.T. SHEN AND X.Y. YAO, Nontrivial solutions for quasilinear elliptic equations with natural growth, Acta Math. Sinica (Chin. Ser.) 52 (2009), 785-798.

[8] Z.X. Li, Y.T. Shen AND Y.M. ZhANG, An application of nonsmooth critical point theory, Topol. Methods Nonlinear Anal. 35 (2010), 203-219.

[9] S.I. PohožAev, Eigenfunctions of $\Delta u+\lambda f(u)=0$, Soviet Math. Dokl. 6 (1965), 14081411.

[10] Y.T. SHEN, Nontrivial solution for a class of quasilinear equation with natural growth, Acta Math. Sinica (Chin. Ser.) 46 (2003), 683-690.

[11] M. Squassina, Spike solutions for a class of singularly perturbed quasilinear elliptic equation, Nonlinear Anal. 54 (2003), 1307-1336.

[12] W. Zou, Sign-Changing Critical Point Theory, Springer, 2008.

YAOTIAN Shen AND Youjun WANG

Department of Mathematics

South China University of Technology

Guangzhou 510640, P.R. CHINA

E-mail address: maytshen@scut.edu.cn, scyjwang@scut.edu.cn

ZHOUXIN Li

School of Mathematics and Statistics

Central South University

Changsha 400083, P.R. CHINA

E-mail address: lzx@math.pku.edu.cn

TMNA : Volume $43-2014-\mathrm{N}^{\circ} 2$ 